\title{
COVID-19 disease information management model to reduce the negative impact on the infodemics of the university community
}

\author{
Flor B. Montañez, Alberto L. Morán, Victoria Meza-Kubo
}

Published: 30 November 2021

\begin{abstract}
The COVID-19 disease has put official health institutions on alert. They are aware of the problem of infodemics and the negative impact it has on health emergencies, and of the need to disseminate objective, complete and useful information on COVID-19 disease to all types of audiences. These have been supported by the different existing media and information systems that allow them to manage the infodemics. For this reason, this research topic proposes to define a model to manage information on COVID-19 disease, through a screening process (selecting, verifying, validating and presenting information) to deliver information in customized formats for specific audiences and reduce the negative impact of infodemics.
\end{abstract}

\section{Keywords:}

Infodemic; COVID-19; Information management; Information systems; Misinformation.

\section{Introducción}

El COVID-19 es una enfermedad similar a otros coronavirus ya conocidos como el MERS y el SARS [15], pero debido a su evolución, adaptación y propagación ha sido motivo de preocupación mundial. Debido a esto la OMS (Organización Mundial de la Salud) la ha declarado como una emergencia de salud pública de importancia internacional [15].

La enfermedad del COVID-19 se ha convertido en un fenómeno de gran magnitud y relevancia, que ha tenido un impacto en áreas como: salud, sociedad, economía, educación, medios de comunicación, etc. Sin embargo, todas estas áreas para seguir adelante han tenido que modificar sus actividades y adaptarse a medida que avanza este fenómeno. Para lograrlo es necesario que se mantengan informadas y actualizadas.

Uno de los problemas generados por la enfermedad del COVID-19 es el exceso de información y desinformación en el área de la salud. Este problema aumenta considerablemente cuando suceden hechos de preocupación mundial; donde debido al exceso de información se crea un entorno de incertidumbre a través de una

Montañez, Flor B., Morán, Alberto L., Meza-Kubo, Victoria Facultad de Ciencias, Universidad Autónoma de Baja California (UABC)

Ensenada, Baja California, México.

\{flor.montaez, alberto.moran,mmeza\}@uabc.edu.mx mayor participación de las personas. Ocasionando la creación de información con diferentes características y niveles de credibilidad, motivando diferentes puntos de vista sobre la misma información. Provocando que las personas presenten una resistencia en la adopción de las medidas de control que promueven las autoridades de salud. Este problema puede originar en las personas miedo, pánico social, ansiedad o nerviosismo; lo cual, repercute directamente en las medidas de prevención, la propagación, el tratamiento, la recuperación y finalmente en la salud mental durante el tiempo de aislamiento de la enfermedad COVID-19 [5].

De esta manera, durante las epidemias es relevante obtener información confiable, que sea útil para la persona que la necesita y en el momento que la necesita para ayudar a tomar decisiones informadas. Por esta razón, es imprescindible la existencia de información oportuna, suficiente, transparente y de base científica, que sea difundida a través de medios de información confiables. Así mismo, es necesario que la información sea presentada en formatos de información adecuados, para que las diferentes audiencias comprendan y apliquen dicha información en su vida. Esto puede lograrse a través de la aplicación de las Ciencias de la Información, al investigar las propiedades, comportamientos y métodos para procesar y presentar la información a través de técnicas de procesamiento y sistemas de información [2]. Este conjunto de características y procesos por los que pasa la información pueden plasmarse dentro de un modelo para la Gestión de la Información. De esta manera, un modelo de Gestión de la Información proporcionará una representación de los elementos que permitan recabar, procesar y presentar información confiable sobre la enfermedad COVID-19, en audiencias específicas.

Considerando lo anterior, se identifica la necesidad de establecer un modelo que permita gestionar la información sobre la enfermedad COVID-19, para la entrega de información específicamente en la comunidad universitaria y lograr disminuir el impacto negativo que genera la infodemia en ellos. Proponiendo y utilizando un proceso para tamizar la información; es decir, que permita seleccionar, filtrar, validar, identificar aspectos importantes del tema y presentar la información en formatos personalizados para las audiencias específicas; logrando que las personas a través del conocimiento adquirido puedan aplicarlo en su vida de manera confiable.

\section{Antecedentes}

La información sobre el nuevo coronavirus se ha difundido ampliamente por los diferentes medios de comunicación. Las 
organizaciones de salud pública como la OMS, se han encargado de vigilar la pandemia y publicar información actualizada en sus sitios web; desde los síntomas, medidas de prevención, propagación, atención médica y datos estadísticos. Sin embargo, todos los medios de comunicación se han visto superados por el exceso de información y la desinformación, y han buscado la manera de difundir información oportuna, transparente y de base científica acerca de la enfermedad para fomentar la confianza de las personas. Por ello, se han apoyado en todos los recursos existentes y disciplinas científicas.

Existe una disciplina científica llamada infodemiología, encargada de abordar las necesidades de elaboración de políticas de salud, generando pruebas para el desarrollo de herramientas, métodos, y gestión de la infodemia, y contribuir en la vigilancia de brotes epidémicos [16]. Es a través de la infodemiología que se puede estudiar y abordar la infodemia. El término de infodemiología fue acuñado en el año 2002 por el investigador Gunther Eysenbach, al identificar que la mayoría de la información de salud que se difunde en los medios sociales e Internet durante una epidemia, no concuerda con la evidencia. Llamando así a los métodos de identificación, estudio y manejo de la desinformación como infodemiología [6]. En 2003, el periodista y experto en política David Rothkopf durante la epidemia del SARS, identificó la presencia de una epidemia de información que aumentó y prolongó el daño social, económico y de salud pública. Nombrándola como "Infodemia" y definiéndose como "la información asociada con miedo, especulación y rumores, amplificada y transmitida rápidamente por las modernas tecnologías de la información y que afecta negativamente la economía, la política y la seguridad de manera desproporcional a la realidad" [14]. Más tarde en 2011, nuevamente el investigador Eysenbach publica una definición formal sobre infodemiología, definiéndose como "la ciencia de la distribución de los determinantes de la información en un medio electrónico, específicamente Internet o en una población, con el objetivo de informar sobre la salud y la política pública"; además de explicar su relación con algunos conceptos clave [7]. En 2018, la OMS utiliza el concepto de Infodemia en su libro "Manejo de epidemias" [18]. Por último, a inicios del año 2020 es cuando el término de Infodemia se ha vuelto de interés de estudio, debido a la pandemia ocasionada por la enfermedad COVID-19. Además, el 15 de febrero del 2020 el director de la OMS Tedros A. Ghebreyesus declara que hay una "epidemia de información" y que "No sólo estamos luchando contra una epidemia; estamos luchando contra una infodemia" [19]. Debido a este suceso, la infodemia se refiere a un gran aumento del volumen de información y desinformación relacionada con un tema particular de salud, que puede volverse exponencial en un período corto debido a un incidente concreto [3], como es el caso de la enfermedad COVID-19.

La desinformación puede estar compuesta por información errónea, falsa o engañosa, su transmisión se realiza entre las personas que desconocen cómo interpretar la información que es verídica [1], provocando un impacto negativo en las personas. Debido a que la desinformación puede difundirse y asimilarse más rápido y llegar hacer más daño que el hecho de sólo recibir información. Esto nos lleva al impacto negativo que provoca la infodemia en los individuos o en las comunidades; generando consecuencias como: dificultad en encontrar fuentes de información confiables, crear un entorno de incertidumbre, tomar malas decisiones, etc. [9, 20]. Por otro lado, también se refleja en las personas a través de percepciones negativas como: pánico, miedo, desconfianza social, vulnerabilidad, prejuicios que dañan, polarización, potenciar teorías de conspiración, etc. [4].

\section{Trabajo relacionado}

Con la intención de identificar el trabajo que se está realizando para ayudar a disminuir el impacto negativo de la infodemia en audiencias específicas, se realizó una revisión sistemática de literatura siguiendo la metodología propuesta en [12]. Las bases de datos utilizadas fueron ACM, EBSCO, Elsevier, IEEE y Wiley. Para los criterios de inclusión se consideraron trabajos que presentaran sistemas de información, trabajos que hablaran sobre la infodemia o desinformación generada por la enfermedad COVID19, trabajos que fueran presentados entre los años 2002 y 2021, entre otros. Se obtuvieron 914 artículos en total, de los cuales se eliminaron duplicados y aquellos que no cumplían con los criterios de inclusión, quedándonos con sólo 48 artículos.

Entre los resultados de la revisión se tiene que, las diversas organizaciones, instituciones y sistemas de información tienen presente el problema de la infodemia que se genera en emergencias de salud internacionales y de la necesidad de poder transmitir información veraz, confiable y útil para todo tipo de audiencias sobre la enfermedad COVID-19. Algunos de los sistemas y organizaciones han llevado a cabo su propio proceso para seleccionar, filtrar, validar y presentar la información. Si bien, existen herramientas tecnológicas que pueden ayudar a disminuir la infodemia o desinformación sobre diversos temas. La pandemia ocasionada por la enfermedad del COVID-19 amplía el área de oportunidad debido a la importancia de contar con información confiable, relevante y oportuna en formatos que sean fácilmente comprensibles por los usuarios. Además, considerando la cantidad y la frecuencia con que aparece nueva información sobre el tema, hace necesario que surjan herramientas que apoyen en esta tarea.

Por esta razón, sería útil contar con un modelo que permita gestionar la información de la enfermedad COVID-19, a través de un proceso para tamizar la información, y presentarla en formatos adecuados para cada audiencia, con el objetivo de ayudar a disminuir el impacto negativo de la infodemia específicamente en la comunidad universitaria. El modelo de gestión de la información debe considerar criterios de calidad de la información, elementos para un proceso de tamizaje y presentación de la información. Se debe fundamentar en el conocimiento de los profesionales de la salud, para proporcionar información confiable sobre el área clínica $\mathrm{y}$ en los profesionales de la comunicación para adecuar la información a un lenguaje y formato comprensible. También, debe incluir las preferencias de información de los consumidores, para que obtengan conocimiento acerca de la enfermedad COVID-19 y puedan aplicarlo en su vida de manera confiable.

Finalmente, el modelo de gestión de la información se podría emplear como una herramienta para el entendimiento de la infodemia sobre la enfermedad COVID-19 y el impacto que genera en los individuos o comunidades. También, como una herramienta de diseño utilizada para la presentación de información en diferentes formatos, con base en las preferencias de una audiencia y en los criterios de calidad de la información. Del mismo modo, podría emplearse para el desarrollo de sistemas de información que ayuden a disminuir el impacto en la infodemia. Además, al adecuar el tipo de información se podría contextualizar para otros temas y comunidades específicas.

\section{Descripción de la investigación}

Con base en el problema descrito anteriormente, se definió la siguiente pregunta que guía la investigación: ¿Cuál es el impacto de gestionar la información sobre la enfermedad COVID-19 en la infodemia de la comunidad universitaria? Para dar respuesta a esta pregunta, se han derivado las siguientes: 
- ¿Cuáles son las principales necesidades y preferencias de información en la comunidad universitaria, acerca de la enfermedad COVID-19?

- ¿Cuáles son y cómo se pueden medir las variables que permitan determinar un cambio en el impacto negativo en la infodemia de la comunidad universitaria?

- ¿Cuáles son los elementos necesarios que se deben considerar para establecer y definir un proceso para la gestión de la información de la enfermedad COVID-19?

- ¿Cómo se puede modelar el proceso de gestión de la información para que sea de utilidad a los expertos del área?

- ¿Cuál es el efecto en la infodemia de la comunidad universitaria después de gestionar la información de la enfermedad COVID-19, a través de una implementación del modelo?

\subsection{Objetivo de la investigación}

El objetivo general de la investigación es: Definir y evaluar un modelo de gestión de la información para la entrega de información en la comunidad universitaria, acerca de la enfermedad COVID-19 y medir el impacto negativo en la infodemia.

\subsection{Variables}

Se propone que la variable independiente sea medida a través de la calidad de la información. La cual, está determinada por la capacidad de satisfacer las necesidades de información de la persona que la consulta; es decir, está condicionada a la valoración subjetiva del consumidor. Sin embargo, se han identificado trabajos como el presentado en [17], donde los autores a partir de la necesidad de evaluar la información o los datos en términos cuantitativos, establecen una serie de dimensiones con diferentes criterios (como la objetividad, integridad, actualidad, utilidad, etc.). Para medir la calidad de forma objetiva, es necesario descomponerla en características observables llamadas dimensiones de calidad, a través de las cuales es posible definir, identificar y medir desde un determinado objetivo [10]. Es importante mencionar que no existe un conjunto de dimensiones universales, cada autor es libre de definirlas y adaptarlas a su contexto. Por esta razón, se propone medir la variable independiente a través de una lista de criterios de calidad de la información, que permitan determinar la veracidad de la información y la calidad de la presentación de la información. La veracidad es la que ayuda a corroborar si la información es verdadera o falsa, al contrastar los criterios de: actualidad de la información, autoría, integridad, precisión, objetividad, utilidad, pertinencia, contextualidad y adecuación al usuario. Mientras que, para la calidad de la presentación puede ser a través de: el tipo de medios de comunicación, las fuentes de información, tipo de formato, documento, estructura, diseño y originalidad.

En cuanto a la variable dependiente, se propone que sea el impacto negativo en la infodemia de la comunidad universitaria sobre el tema de la enfermedad COVID-19. La cual, estará determinada por los criterios de la veracidad y la calidad de la presentación de la información.

\subsection{Hipótesis}

Se plantean dos hipótesis para determinar si los criterios de calidad de la información, en cuanto a la veracidad y presentación ayudarán a disminuir el impacto negativo de la infodemia en la comunidad universitaria. Entendiendo que la disminución del impacto en la infodemia es controlada por el incremento en la información (veracidad y en la calidad de la presentación) y la disminución en la desinformación; el cual se verá reflejado en la medición de las percepciones negativas

H1: El incremento en la veracidad de la información, disminuirá el impacto negativo en la infodemia de la comunidad universitaria sobre la enfermedad COVID-19.

H2: El incremento en la calidad de la presentación de la información, disminuirá el impacto negativo en la infodemia de la comunidad universitaria sobre la enfermedad COVID-19.

\subsection{Metodología de la investigación}

La metodología consiste de 3 etapas: 1) Entendimiento, 2) Pruebas de concepto y 3) Elaboración del modelo (ver Figura 1). Las etapas están basadas en la metodología para el diseño centrado en el usuario [13] y adaptadas de las metodologías propuestas para la elaboración de ontologías, consideradas para modelos formales [8, 11]. Agregando las características de iterar y trabajar de manera simultánea entre las etapas. Para cumplir el objetivo de la investigación se propone realizar tres iteraciones a la metodología.

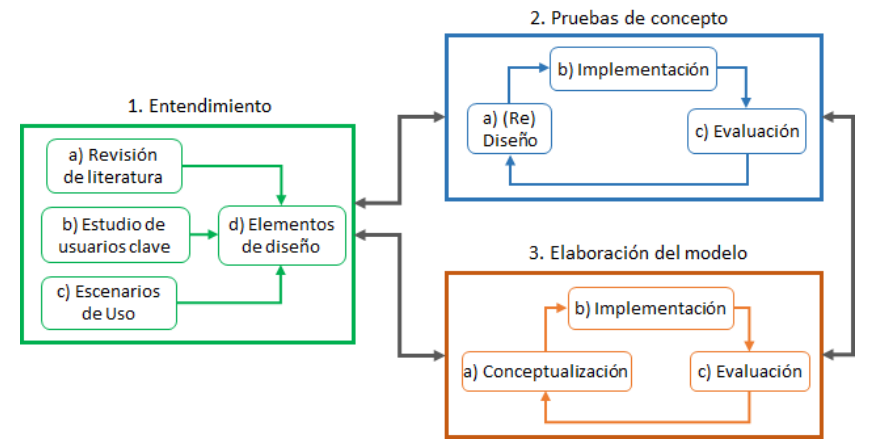

Figura 1. Metodología para el trabajo de investigación.

\subsubsection{Descripción de las etapas}

La etapa de Entendimiento consiste en obtener información sobre las características y criterios que debe cumplir la información, los elementos para el proceso de gestión de la información y las características de diseño para los formatos de presentación. Por medio de estudios a los profesionales de la salud, de la comunicación y a la comunidad universitaria para plasmar escenarios de uso sobre situaciones reales. Además de clarificar la necesidad de establecer un modelo el propósito, el contexto, los objetivos y los requisitos de la investigación. En la etapa de Pruebas de concepto se deben diseñar e implementar prototipos de sistemas de información que integren los elementos de diseño identificados en la etapa 1 . Se deben realizar evaluaciones a los prototipos desarrollados y considerar los resultados de las evaluaciones como retroalimentación para desarrollar prototipos incrementales que cumplan las características iniciales. Por último, en la etapa Elaboración del modelo se busca estructurar y organizar el conjunto de conocimiento proveniente de la etapa de Entendimiento y los resultados obtenidos de las evaluaciones de los prototipos. Generar versiones del modelo de gestión de la información para realizar representaciones formales mediante un lenguaje para modelos. Y realizar verificaciones de forma iterativa en cuanto al cumplimiento de los objetivos de la investigación. Realizar validaciones con especialistas del área, en cuanto a los factores identificados de acuerdo al lenguaje elegido para su implementación, el entendimiento y dominio del tema; con el objetivo de retroalimentar el modelo propuesto. El impacto negativo de la infodemia es analizado en las tres etapas de la metodología. En la primera desde un punto de vista teórico; en la segunda, a través de estudios cualitativos con el objetivo de contrastar lo identificado en 
la literatura; por último, en la tercera etapa se busca medir si el modelo propuesto el útil para disminuir ese impacto negativo.

\section{Cuestiones y retos a discutir}

Algunos puntos que sigo explorando es tener conocimiento de otras propuestas de modelos de gestión de la información sobre temas de salud que implemente algún proceso para filtrar información, de manera que estos puedan ser tomados como referencia para la investigación. Así, como sistemas de información que implementen procesos para filtrar, verificar y/o presentar la información.

Uno de los retos de mi trabajo es cómo medir el impacto de la infodemia y como evaluar la pertinencia de la información presentada en formatos adecuados a audiencias específicas.

\section{Conclusiones}

Con base a los primeros resultados, se está trabajando en la obtención de elementos de diseño para proponer un proceso para la gestión de la información sobre la enfermedad COVID-19. También, se ha propuesto una primera versión de las variables que permitan determinar un cambio en el impacto negativo en la infodemia de la comunidad universitaria, con las cuales se está trabajando en su validación. Con estos resultados se espera poder generar una primera versión del modelo de gestión de la información, para posteriormente evaluarlo a través de pruebas de concepto.

\section{Referencias}

[1] Alfonso-Sánchez I, Fernández-Valdés M. (2020). Comportamiento informacional, infodemia y desinformación durante la pandemia de COVID-19. Anales de la Academia de Ciencias de Cuba, 10(2), 882.

[2] Borko, H. (1968). Information science: what is it? American documentation, 19(1), 3-5.

[3] Cinelli, M., Quattrociocchi, W., Galeazzi, A., Valensise, C. M., Brugnoli, E., Schmidt, A. L., \& Scala, A. (2020). The COVID-19 social media infodemic. Scientific Reports, 10(1), $1-10$.

[4] Depoux, A., Martin, S., Karafillakis, E., Preet, R., WilderSmith, A., \& Larson, H. (2020). The pandemic of social media panic travels faster than the COVID-19 outbreak. Journal of Travel Medicine, 27(3), 1-2.

[5] Durodolu, O. O., \& Ibenne, S. K. (2020). The fake news infodemic vs information literacy. Library Hi Tech News incorporating Online and CD Notes, 37(7), 13-14.

[6] Eysenbach, G. (2002). Infodemiology: The epidemiology of (mis) information. The American journal of medicine, 113(9), 763-765.

[7] Eysenbach, G. (2011). Infodemiology and infoveillance: tracking online health information and cyberbehavior for public health. American journal of preventive medicine, 40(5), S154-S158.
[8] Fernández-López, M., Gómez-Pérez, A., \& Juristo, N. Methontology: from ontological art towards ontological engineering. Proc. AAAI Spring Symp. (1997), 33-40.

[9] Lazer, D. M., Baum, M. A., Benkler, Y., Berinsky, A. J., Greenhill, K. M., Menczer, F. \& Zittrain, J. L. (2018). The science of fake news. Science, 359(6380), 1094-1096.

[10] Lee, Y. W., Strong, D. M., Kahn, B. K., \& Wang, R. Y. (2002). AIMQ: a methodology for information quality assessment. Information \& Management, 40(2), 133-146.

[11] Noy, N. F., \& McGuinness, D. L. Ontology development 101: A guide to creating your first ontology. Technical Report SMI-2001-0880, Stanford Medical Informatics (2001).

[12] Petersen, K., Feldt, R., Mujtaba, S., \& Mattsson, M. (2008). Systematic mapping studies in software engineering. In 12th International Conference on Evaluation and Assessment in Software Engineering (EASE), 12, 1-10.

[13] Rogers, Y., Sharp, H., \& Preece, J. (2011). Interaction design: beyond human-computer interaction (3rd ed.). John Wiley \& Sons, Ltd.

[14] Rothkopf, D. J. (2003). When the Buzz Bites Back. The Washington Post, 11, B1-B5. Recuperado de: https://www.washingtonpost.com/archive/opinions/2003/05/ 11 /when-the-buzz-bites-back/bc8cd84f-cab6-4648-bf58$0277261 \mathrm{af} 6 \mathrm{~cd} /$

[15] Sohrabi, C., Alsafi, Z., O'neill, N., Khan, M., Kerwan, A., Al-Jabir, A., ... \& Agha, R. (2020). World Health Organization declares global emergency: A review of the 2019 novel coronavirus (COVID-19). International journal of surgery, 76, 71-76.

[16] Tangcharoensathien, V., Calleja, N., Nguyen, T., Purnat, T., D’Agostino, M., Garcia-Saiso, S., ... \& Briand, S. (2020). Framework for Managing the COVID-19 Infodemic: Methods and Results of an Online, Crowdsourced WHO Technical Consultation. Journal of Medical Internet Research, 22(6), e19659.

[17] Wang, Y. W. and R. Y. (1996). Anchoring Data Quality Dimensions in Ontological Foundations. COMMUNICATIONS OF THE ACM, 39(11), 86-95.

[18] World Health Organization. (2018). Managing epidemics: key facts about major deadly diseases. Geneva: World Health Organization. Recuperado de: https://apps.who.int/iris/handle/10665/272442

[19] Zarocostas, J. (2020). How to fight an infodemic. The Lancet, 395(10225), 676.

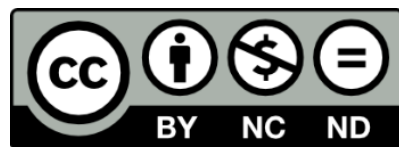

(C) 2021 by the authors. This work is licensed under the Creative Commons AttributionNonCommercial-NoDerivatives 4.0 International License. To view a copy of this license, visit http://creativecommons.org/licenses/by-nc-nd/4.0/ or send a letter to Creative Commons, PO Box 1866, Mountain View, CA 94042, USA. 